\title{
Lugano Classification Limited Stage Adult Lymphoma AJCC v8
}

National Cancer Institute

\section{Source}

National Cancer Institute. Lugano Classification Limited Stage Adult Lymphoma A/CC v8. NCI Thesaurus. Code C141149.

Limited stage adult lymphoma based on the Lugano classification criteria. It includes stages I, IE, II, and IIE. (from AJCC 8th Ed.) 